\title{
CONOCER A LAS FAMILIAS DEL ALUMNADO DE FORMACIÓN PROFESIONAL, UN RETO PARA LA INVESTIGACIÓN EDUCATIVA
}

\author{
Soledad García-Gómez \\ Rosario Ordóñez-Sierra \\ Universidad de Sevilla \\ Rosario Izquierdo Chaparro \\ Ayuntamiento de Dos Hermanas (Sevilla)
}

\begin{abstract}
RESUMEN: Las familias del alumnado de formación profesional apenas son objeto de estudio por parte de la investigación educativa. Pasar de la educación secundaria obligatoria a la formación profesional es una transición en la cual "desaparecen" las familias de los estudiantes. Ser mayores de 16 años, la finalización de la escolaridad obligatoria y la elección de una vía profesionalizante en lugar del bachillerato, son factores que parecen alejar al sistema educativo de las familias y viceversa. Así pues, el objetivo de este trabajo ha sido explorar mediante una metodología cualitativa cuáles son los rasgos descriptivos básicos de familias de este tipo de alumnado. Han participado veinte familias de zonas rurales de la provincia de Sevilla. El análisis de contenido de las entrevistas semiestructuradas destaca la preeminencia de la familia nuclear tradicional, el bajo nivel de cualificación académica y laboral de estas madres y estos padres, así como unas condiciones económicas precarias. Destaca el interés que manifiestan por la formación de sus descendientes y por su futuro laboral. Como principal conclusión resaltar la necesidad de avanzar en esta línea de investigación desde una perspectiva inclusiva con este colectivo de familias, lo cual tendrá repercusiones positivas en el alumnado de formación profesional.
\end{abstract}

PALABRAS CLAVE: Familia, alumnado, formación profesional, investigación cualitativa.

\section{EXPLORING VOCATIONAL EDUCATION AND TRAINING STUDENTS' FAMILIES, A CHALLENGE FOR EDUCATIONAL RESEARCH}

\footnotetext{
ABSTRACT: Vocational Education and Training students' families are not a core for educational research. In the transition from secondary comprehensive
} 
education to VET it seems like families become removed from schools. The reasons for that can be the age of students (older than sixteen years old), the end of comprehensive education and also the VET choice leaving the more academic educational pathway. The aim of our study has been the exploration through a qualitative methodology of the main features that describes these families to better know them. Participants were twenty families living in rural areas around Seville city. Half-structured interviews content analysis show the pre-eminence of the traditional nuclear family, parents' low academic and labour qualification level and their precarious economic conditions. We emphasize that parents are very interested in their children education and future employment. A main conclusion is that it is necessary to do more research on this topic in order to be more inclusive with these families in the educational system. This shall be positive for VET' students.

KEYWORDS: Family, students, vocational education and training, qualitative research.

Recibido: 10/10/2016

Aceptado: 05/04/2017

Correspondencia: Soledad García Gómez, Universidad de Sevilla, Facultad de Ciencias de la Educación, C/ Pirotecnia, s/n, 41013 Sevilla. Email: solgar@us.es.

\section{INTRODUCCIÓN}

La formación profesional en el marco del sistema educativo español cuenta con una dilatada trayectoria. Su evolución ha estado vinculada, sobre todo, a los cambios legislativos. Varios estudios de carácter histórico describen y analizan el camino recorrido (Marhuenda-Fluixá, Salvá, Navas y Abiétar, 2015; Merino, 2005), mostrando cómo a lo largo de todos estos años se ha ido configurando un sistema de formación profesional (FP) que, a pesar de haber ido avanzando, aún no está plenamente asentado, carece de una identidad clara y afronta dificultades de diversa índole (Planas, 2012). En el informe sobre la situación de la formación profesional en España, Homs (2008) se hace eco de esta singularidad y afirma:

A caballo del sistema de educación, el mercado de trabajo y el sistema productivo, la formación profesional aglutina tensiones, aspiraciones, decisiones, contradicciones y tendencias de gran variabilidad que exigen una gran capacidad de síntesis y de información para conseguir entender sus principales características y su evolución de futuro (p. 9).

Esta situación no es exclusiva de España, es compartida tanto en el entorno europeo (Psifidou, 2014) como en otras latitudes como Australia (Chappell, 2003).

En particular, a tenor de la última regulación legislativa (LOMCE, 2013), la formación profesional en el marco del sistema educativo español comprende varias modalidades: Formación Profesional Básica (FPB), Ciclos Formativos de Grado Medio (CFGM) y Ciclos Formativos de Grado Superior (CFGS). El sistema público atien- 
de actualmente a dos tercios del total (MECD, 2016), impartiendo estas enseñanzas en los Institutos de Educación Secundaria (IES), junto a la secundaria obligatoria, el bachillerato y/o la educación secundaria para adultos. Así pues, están integradas formalmente en la vida cotidiana de estos centros, aunque constituyan en la mayoría de las ocasiones una subcultura específica (Lorente, 2006), cuya visibilidad es escasa y/o está difuminada. También se imparten en los Centros Integrados de Formación Profesional que se han constituido en los últimos años.

Esta estructuración de los estudios de formación profesional es particular de nuestro contexto, ya que cada país ha configurado la formación profesional de manera diferente. Greinet (2004: 19) sostiene que el origen de la diversificación se sitúa en la Revolución Industrial, puesto que "el proceso de industrialización en Europa no produjo un único modelo unitario de formación profesional". Según Martínez (2001), en el continente europeo las disparidades entre los sistemas formativos hunden sus raíces en tradiciones históricas diferenciadas en cuanto a la evolución de los respectivos sistemas educativos:

La rama profesional es la predominante para once de los países de la Unión Europea, la dirección es más que evidente para los casos de Alemania, Austria y Holanda, países con sistemas educativos, con una clara tradición profesionalizante. Por contra, y respondiendo también con fidelidad a su propio pasado, encontramos que en Grecia, España, Francia y Portugal son mayoría los estudiantes que prefieren cursar estudios de enseñanza general en lugar de ramas profesionales (p. 254).

A pesar de las diferencias históricas y actuales de los sistemas y modelos de formación profesional en el seno de Europa, la tendencia de futuro parece orientarse hacia la convergencia. Según Psifidou (2014: 359) "existe un consenso entre los países y los interlocutores sociales sobre los aspectos en los que debe centrarse la futura cooperación europea en materia de FP".

En paralelo a la asunción de las medidas que devienen de las directrices políticas nacionales y/o europeas, la formación profesional se ha convertido en objeto de investigación. La revisión de la literatura a nivel internacional permite conocer su configuración actual en los distintos sistemas educativos, además de los principales retos que afronta. De todas formas, se puede afirmar que la formación profesional suele quedar excluida de aquellos estudios que abarcan todo el sistema educativo y/o toda la educación secundaria, la cual se suele restringir a la ESO y al bachillerato. Solo las investigaciones centradas en ámbitos como el fracaso y/o el abandono escolar temprano o la atención a la diversidad la tienen en consideración, por ser destino habitual del alumnado que no cursa bachillerato (Escudero y Martínez, 2012; García, Casal, Merino y Sánchez, 2013).

En un plano más didáctico han visto la luz en los últimos años algunas publicaciones focalizadas en el profesorado (Attwell y Baumgartl, 2008; Misra, 2011) y otras en el alumnado (Cedefop, 2011; Feinstein y Peck, 2008; Renés y Castro, 2013). Sin embargo, el entorno familiar del alumnado de FP es un gran desconocido para la investigación educativa. Apenas se dispone de estudios que indaguen en las familias de este alumnado y, mucho menos, que éstas sean las interlocutoras en los procesos de recogida de datos. En las actas de la International VET-Conference, celebrada en Bremen (Alemania) en septiembre de 2015, se publicaron cuarenta trabajos de veinte países que abordaron conceptos innovadores sobre la formación profesional para el 
siglo XXI (Gessler y Freund, 2015). Entre éstos, solo una contribución hacía alguna referencia a las familias del alumnado de FP. Estamos pues ante un campo de estudio prácticamente inexplorado.

La presencia de las familias del alumnado de FP en la investigación educativa se limita a aquellos trabajos en los que se trazan correspondencias entre los niveles formativos y las ocupaciones laborales de padres y madres con el rendimiento académico de sus hijos e hijas. Ianelli (2002), en un estudio comparado entre doce países europeos representativos de las diferencias en el seno de Europa, encontró que el nivel formativo de padres y madres condiciona los logros educativos y de acceso al primer empleo de sus descendientes, aun habiendo significativas diferencias entre los países analizados. En las conclusiones Ilama la atención acerca de la falta de conocimiento que hay sobre el contexto familiar del alumnado. También Schnabel, Alfeld, Eccles, Köller y Baumert (2002) Ilevaron a cabo dos estudios longitudinales en Alemania y EE.UU., para comparar cómo incide el estatus socioeconómico de los padres en las decisiones de los estudiantes, con respecto a los estudios a acometer y a la institución donde cursarlos. Según los resultados obtenidos, afirmaron que la situación de los padres condiciona las decisiones académicas y vocacionales de sus hijos e hijas. En Australia, Foley (2007) realizó una investigación con todos los estudiantes que acudían al sistema público en 2001, confirmando el vínculo entre el bajo estatus socioeconómico de las familias y la elección de estos estudios.

En las líneas anteriores se ha tratado de mostrar someramente que existe una importante laguna en la investigación en el ámbito de la formación profesional. Se desconoce cómo son los contextos familiares de este alumnado y qué opinan estas familias acerca de los estudios de formación profesional, los cuales, según datos del MECD (2016), están en alza (en una década se ha pasado en España de 454.053 estudiantes a 767.528).

\section{Proceso metodológico}

El propósito general del estudio acometido era conocer las opiniones de las familias de alumnado de formación profesional (en particular de Ciclos Formativos de Grado Medio) sobre la situación de sus hijos e hijas, el desempeño en el ciclo, las expectativas de futuro, así como las dificultades afrontadas y superadas hasta el momento (García-Gómez, Ordóñez-Sierra, Vinuesa y Izquierdo, 2016).

Para ello, se llevó a cabo un estudio de carácter exploratorio, enfocado desde las premisas de la metodología cualitativa encuadrada en un paradigma hermenéutico (Flick, 2007), en la medida en que el interés estribaba en aproximarse a este colectivo de familias de estudiantes para conocerlas. Se buscaba el contacto directo, personal y natural y, para ello, lo más adecuado eran las entrevistas (Kvale, 2011).

\section{El acceso a las familias participantes}

La muestra de partida debía ser intencional, ya que se había seleccionado a un colectivo de personas al cual no es fácil acceder. La población eran madres y padres de alumnos y alumnas que cursaran un CFGM de cualquier familia profesional en un IES de la provincia de Sevilla (España). Se encuadra el estudio pues en el ámbito rural andaluz. 
El principal escollo del proceso era cómo contactar con las familias. Se afrontó el reto con tesón y esfuerzo, al saber que el proceso sería difícil y requeriría tiempo. La estrategia inicial fue contactar con institutos con los que había algún tipo de vínculo previo, para que ellos fuesen los intermediarios entre las familias y las investigadoras. Así pues, la forma de proceder, tras conversaciones y negociaciones telefónicas con los equipos directivos y/o departamentos de orientación de los IES, fue acudir a éstos, presentar el estudio y solicitar la colaboración de los estudiantes para acceder a sus familias. Se les pidió que Ilevaran a casa las cartas que se les entregaron y que solicitaran su colaboración. Conforme se recibían las respuestas, se contactaba vía telefónica con quienes accedían a participar para concertar los encuentros, que tuvieron lugar en varias localidades. La mayoría de las entrevistas se desarrollaron en los domicilios particulares.

En un primer momento se decidió entregar las cartas solo al alumnado de 16 a 18 años, suponiendo que los mayores de edad no serían proclives a participar, como tampoco sus familias. Sin embargo, el profesorado instó a entregarlas a los grupos completos independientemente de las edades. Así se hizo, lo cual ha permitido, para sorpresa de todos, entrevistar a familias de jóvenes de hasta 25 años. Se acudió a 15 institutos de 9 localidades del sur de la provincia de Sevilla (de los 71 que imparten CFGM en 44 municipios), y se repartieron más de 600 cartas. El proceso brevemente descrito terminó con 28 familias aceptantes, de las cuales solo se pudo acceder a 20, que correspondían a 22 estudiantes al haber dos familias con más de un hijo/a cursando un CFGM. Hemos trabajado con los datos de 18 entrevistas, pues dos de ellas apenas aportaban información relevante. En la tabla 1 se aportan algunos datos descriptivos de ellas y de sus hijos e hijas.

Tabla 1. Datos descriptivos de la muestra

\begin{tabular}{|c|c|c|c|c|c|c|c|}
\hline \multicolumn{3}{|c|}{ Familias entrevistadas } & \multicolumn{5}{|c|}{ Ciclos Formativos cursados por los hijos/as de las familias } \\
\hline $\begin{array}{c}\text { Padres } \\
5\end{array}$ & $\begin{array}{c}\text { Madres } \\
10\end{array}$ & $\begin{array}{c}\text { Padre y } \\
\text { madre } \\
3\end{array}$ & $\begin{array}{c}\text { Informática y } \\
\text { Comunicaciones } \\
14 \text { chicos }\end{array}$ & $\begin{array}{l}\text { Auxiliar de } \\
\text { Enfermería } \\
3 \text { chicas }\end{array}$ & $\begin{array}{l}\text { Act. Física y } \\
\text { Deportiva } \\
1 \text { chica }\end{array}$ & $\begin{array}{l}\text { Hostelería } \\
\text { y Turismo } \\
1 \text { chico }\end{array}$ & $\begin{array}{c}\text { Electricidad y } \\
\text { Electrónica } \\
1 \text { chico }\end{array}$ \\
\hline $\begin{array}{l}\text { Edad media padres: } 51 \text { años } \\
\text { Horquilla de edad: } 41 \text { a } 55\end{array}$ & $\begin{array}{l}\text { dia padres } \\
\text { de edad: }\end{array}$ & $\begin{array}{l}51 \text { años } \\
41 \text { a } 55\end{array}$ & \multicolumn{5}{|c|}{$\begin{array}{l}\text { Edad media estudiantes: } 17 \text { años } \\
\text { Horquilla de edad: } 16 \text { a } 25 \text { años }\end{array}$} \\
\hline & & & ntes en 7 loca & es de la & incia de $S$ & & \\
\hline
\end{tabular}

\section{Recogida y análisis de los datos}

Las veinte entrevistas se llevaron a cabo en los meses de octubre y noviembre de 2014. La duración media de las mismas fue de veinticinco minutos. Es de destacar que en muchas ocasiones la conversación finalizaba con unas palabras de los padres y/o las madres agradeciendo que se les hubiera preguntado acerca de sus hijos e hijas. Se daba una mezcla de sorpresa y gratitud por el interés mostrado en conocer sus opiniones. 
El instrumento de recogida de datos se estructuró en tres dimensiones correspondientes a tres etapas en la vida de los chicos y las chicas:

- El "Pasado", que incluía preguntas que atendían a la trayectoria académica y vital en educación primaria y durante la ESO;

- El "Presente", que se centraba en el desarrollo del ciclo formativo: estado anímico de los jóvenes, rendimiento, satisfacción, apoyos, etc.;

- El "Futuro", que giraba en torno a las expectativas vitales, académicas y profesionales de las familias respecto de sus hijos e hijas.

El guion de las entrevistas semiestructuradas incluía también un conjunto de datos sociodemográficos básicos de las personas entrevistadas como: edad, estudios realizados, situación laboral, puesto de trabajo, situación familiar, etc. En el devenir de las conversaciones, las familias iban aportando otras informaciones sobre sus propias vidas que emergieron en el proceso como datos muy relevantes. Aludían a la precaria economía doméstica, a su situación laboral, al motivo de abandono de sus estudios o a la propia estructura familiar. Por ello, a pesar de no ser un objetivo inicial de la investigación, a la luz del interés de los datos recabados se optó por analizarlos y divulgarlos a través de este artículo. Se podría decir, pues, que el objetivo específico del análisis que se presenta ha sido describir el contexto familiar de algunos alumnos y alumnas de CFGM a partir de la información aportada por las propias familias. Los datos que las describen han sido agrupados en varias dimensiones para su análisis, dando lugar a un sistema de categorías, las cuales se identifican con los siguientes códigos:

- estructura familiar (FAM);

- estudios y/o situación laboral de madres y padres (LAB);

- relativo a la formación de sus descendientes (FOR);

- expectativas sobre el futuro de hijos e hijas (EXP);

- otros aspectos de su vida cotidiana: cuidado de familiares mayores, atención a los menores (VID).

Tras el pertinente trabajo de transcripción, reducción de datos y codificación (Rodríguez, Gil y García, 1996), se procedió al análisis de aquellos con la ayuda del programa informático Atlas.ti (versión 6.2). Los párrafos textuales que se incluyen en el apartado siguiente van acompañados de un código extenso que aporta información sobre la temática (códigos ya reseñados) y sobre quién lo ha enunciado: número de orden de la entrevista, seguidos de $\mathrm{M}$ o $\mathrm{P}$ según sean palabras de la madre o del padre, más O o A según el alumno sea chico o chica. Ejemplo: (LAB08MO), corresponde a un fragmento sobre la situación laboral de la madre de un chico, entrevista número ocho. La abundancia de fragmentos provenientes de las madres revela que son éstas quienes han mencionado más cuestiones familiares.

\section{Resultados}

Los resultados que se presentan no son representativos del total de la población ya que no derivan de una investigación concebida a tal efecto. Se limitan a los casos particulares analizados que, a pesar de no ser generalizables, se consideran de gran valor. 
El tipo de familia más frecuente entre los entrevistados (a partir de los datos clasificados en la categoría FAM) es la familia nuclear (padre y madre) con dos hijos (las edades de los hermanos y hermanas oscilan entre los 11 y los 27 años de edad). Aparte de las familias nucleares hay una familia reconstituida y dos parejas que se han separado.

Como se recoge en la tabla 1, la edad media de los padres es de 51 años, siendo las madres algo más jóvenes (edad media 48 años). Por tanto, se habla de adultos que casi todos han nacido en la década de los sesenta del siglo XX.

A nivel general se puede afirmar que el nivel de estudios de los padres y de las madres es bajo (datos categoría LAB). Solo uno de los padres ha realizado estudios universitarios, es maestro. Los demás obtuvieron el graduado escolar o solo el certificado de escolaridad si abandonaron antes de terminar la Educación General Básica (estaba en vigor la Ley General de Educación de 1970). Pocos padres han realizado estudios de FP.

En el caso de las madres la situación es similar, quizás se podría decir que levemente mejor pues la mayoría terminó la EGB. En muchos casos el motivo de abandono de la escuela fue la necesidad de trabajar para ayudar económicamente a sus familias. Este hecho les influye a la hora de incitar a sus hijos e hijas a continuar formándose.

"Yo siempre he estado trabajando desde los ocho años, dejé muy pronto los estudios. Y mi marido también". (LAB12MO)

"Me acuerdo que cuando yo era pequeño mi padre me decía: estudia y prepárate; pero, claro, en aquellos entonces pues hacía falta dinero en la casa y teníamos que trabajar. Y sacamos la EGB y enseguida estábamos trabajando, yo he estado trabajando y estudiando, las dos cosas". (LAB01PO)

Con respecto a las ocupaciones laborales actuales de los padres hay variedad de circunstancias pero, en todo caso, cuando no están desempleados están desarrollando trabajos de baja cualificación profesional. El más habitual es el que se desempeña en el campo, dado el ámbito rural y agrícola donde vive la mayoría de las familias participantes en el estudio. Otras ocupaciones son: chófer, albañil, pintor, vigilante de seguridad y mecánico. Las madres se declaran amas de casa y aquellas que también trabajan fuera del hogar lo hacen como operarias agrícolas, limpiadora, monitora infantil, administrativa u operaria de fábrica.

Los padres y las madres entrevistados, en general, están viviendo situaciones laborales y económicas difíciles, bien porque se encuentran en desempleo bien porque desempeñan trabajos en condiciones precarias.

"Yo siempre estoy de limpiadora o de mantenimiento, a lo mejor trabajo una vez en el año 6 meses o 3 meses, dependiendo de cómo esté la cosa". (LAB07MO)

"Eso no lo ha dicho ella nunca [montar su propia empresa], porque como sabe que en mi casa dinero no hay... Eso es muy complicado, porque aquí lo que tenemos mi marido y yo es el campo, cuando hay, y yo echando horas limpiando... Entonces dinero para eso no hay". (LAB16MA)

Estas circunstancias condicionan a sus descendientes, hay quienes se sienten obligados a colaborar con la precaria economía familiar, y quienes ven frustradas sus aspiraciones ante la falta de medios económicos que sostengan sus proyectos de futuro. 
" No tengo trabajo ni yo! Mira, tengo cinco promociones pendientes y todavía no ha salido ni una. A él le afecta, hombre, yo nunca muestro que estoy preocupado ni mucho menos, yo soy una persona que lo mismo estoy riendo..., pero en este tema no, a él le gustaría venirse conmigo, trabajar y demás, porque él quiere aportar algo en la casa". (LAB14PO)

"Él se quería ir a Montellano o a Sevilla, ya sabes, la edad, pero yo le dije que no, que no se podía, que nosotros no podemos. Porque nosotros ahora mismo estamos parados y yo no puedo, es que no podemos, que hiciera algo aquí". (LAB12MO)

Los problemas laborales que están padeciendo condicionan su percepción de las dificultades que sus hijas e hijos tendrán que afrontar para avanzar en sus estudios y para la posterior incorporación al mercado laboral.

"Estos dos años de paro le han servido a mi hijo para darse cuenta de que tiene que estudiar. Se ha arrepentido bastante de no terminar sus estudios, y la novia lo está motivando mucho (...) Pero menos mal que se ha dado cuenta. Yo se lo decía: ¡mira cómo estamos nosotros! ¿Tú quieres estar como nosotros?". (FOR11MO)

Les animan a formarse siendo muy conscientes de cuáles son las limitaciones que han de sortear. Valoran los estudios que realizan sus descendientes y los contraponen, por experiencia propia, a la incorporación temprana al trabajo y a la dureza de las labores agrícolas.

"Siempre le he machacado a él diciéndole que se preparara, que cuando tuviera edad de prepararse que se preparara, porque el trabajo siempre ha estado malo, no es de ahora, el trabajo siempre ha estado malo en todos los tiempos y, claro, no es lo mismo una persona que esté preparada a una que no esté preparada". (FOR01PO)

"Él sabe lo importante que es tener estudios, que yo me lo he llevado por la tarde a echar unas horitas en el campo, pero para que vea que no es fácil". (FOR12MO)

Una de las madres comenta cómo, cuando su hijo empezó a decir que iba a abandonar el ciclo formativo, decidió que éste trabajase en las faenas agrícolas para que valorase los estudios y cambiase de idea. Fue su estrategia para contraponer una y otra opción.

"Y ya el año pasado él no quiso seguir por las navidades, y estuve hablando con la maestra y demás $y$, bueno, dije: que coja aceitunas y que sepa también lo que es el campo". (LAB06MO)

Estas familias persiguen una movilidad social ascendente por la vía de la educación, muestran su deseo de que sus hijos e hijas alcancen un nivel académico más alto que el que ellos y ellas consiguieron. En algunos casos, el que vayan avanzando en sus estudios de formación profesional ya es motivo de satisfacción.

"Su padre incluso más [contento] que yo, porque como él no tiene estudios, no acabó la EGB, se siente muy orgulloso de que su niño haya avanzado un poquito más que él, incluso está más contento que yo". (EXP08MO)

"Yo siempre les he dicho que tienen que hacer algo, estudiar todo lo que puedan y más. En la familia no hay ninguno que tenga carrera, todos estamos trabajando. Ella es la única que hasta ahora quiere seguir estudiando, y yo lo que quiero es que siga". (EXP19MA) 
A tenor de los testimonios obtenidos, son las madres quienes más se ocupan y preocupan por los estudios de sus hijos e hijas. Suelen ser ellas quienes se encargan de mantener las relaciones con el profesorado, de acudir a las reuniones y de hacer el seguimiento de los estudios.

"Yo siempre me he preocupado, yo más, porque mi marido trabaja fuera. Yo siempre he ido". (FOR05MO)

"Yo sola. Mi marido no ha ido con los dos ni una sola vez al colegio. $\mathrm{Ni}$ a recoger notas, ni a reuniones, mi marido es que no ha pisado el colegio. ¡Y mira si habrán tenido fiestas de fin de curso los dos!". (FOR19MA)

Uno de los padres aludía a la problemática de la incompatibilidad de los horarios laborales y los escolares para explicar por qué no acudía a las reuniones.

"Es que mi trabajo es la pintura y cuando se habla con los profesores son las cuatro de la tarde y esa es hora de trabajo". (FOR11PO)

En el caso de las madres separadas de sus parejas, son ellas quienes conviven con sus hijos e hijas y también quienes mantienen el contacto con los centros educativos.

"Estoy separada y yo soy la que lleva todo el tema de los estudios de ellos". (FOR10MO)

Hay madres y padres que explicitan que su escaso nivel formativo les limita a la hora de ayudarles con las tareas académicas. Dicho problema lo solventan con la ayuda de los hermanos mayores o con clases particulares cuando es posible.

"Yo le digo: no me hables técnicamente, porque nosotros estamos 'cuajaos', por eso viene un profesor que le ayuda y le da clases". (FOR17PO)

"Él se gestiona todo, porque por desgracia yo no he estudiado y ¡tú sabes! él me ha enseñado a mí muchas cosas que yo no sabía, estudiando, repasando, he aprendido muchas cosas que no sabía". (LAB12MO)

"Normalmente cuando el hermano está en casa muchas de las dudas que tienen se las preguntan al hermano, porque el hermano lleva ya mucho tiempo estudiando". (FOR20MA)

En ocasiones, quienes tienen hijos más mayores, cuentan que han tenido problemas para que terminasen los estudios obligatorios. En la mayoría de los casos se han incorporado al mundo laboral o han realizado un ciclo formativo. Solo la hermana de uno de los chicos está cursando estudios universitarios.

[Otro hijo] "Él no llegó ni a terminar la ESO. Él no quería estudiar, él quería trabajar, trabajar... Se echó novia y tiene dos hijos ya. Ahí está, se quitó en $3^{\circ}$ de la ESO y se fue a hacer un curso de electricidad, que es lo que se hacía aquí". (FAM19MA)

"Tengo una hija en la Universidad estudiando Pedagogía". (FOR02MO)

Lo que sí parecen tener claro en general es que los jóvenes deben estudiar aquello que les guste. Parece leerse entrelíneas que temen forzarles a realizar estudios que no desean para evitar que abandonen definitivamente el sistema educativo. También puede ser un reflejo de sus propias trayectorias vitales y laborales, ya que han tenido que ocuparse en empleos no deseados para poder subsistir y mantener a la familia. 
"Nosotros le dijimos: tú lo que vayas a estudiar que te guste y estés a gusto y estés bien y no te sientas obligado por nada". (FOR09PO)

"Cuando es algo que no te gusta, siempre vas a acabar hartándote y dejándolo". (FOR08MO)

"Él tiene que estudiar algo que le guste y se sienta realizado, porque si va a estudiar algo que luego va a trabajar a disgusto..., como que no". (FOR10MO)

En otro orden de cosas destacar que hay familias que en el transcurso de las entrevistas mencionan a los abuelos y a las abuelas, siendo las madres y, en ocasiones, los propios chicos y chicas quienes se ocupan de ellos. Se incorporan textos que los nombran pues aportan información relevante y poco visible de las relaciones familiares.

"Le ha afectado mucho la muerte de su abuela, ella murió el 30 y el examen era el martes, él fue pero no pudo hacer el examen, porque él la quería mucho y me ayudaba a levantarla, a acostarla, a darle los medicamentos. Eso último para él ha sido duro, le ha afectado mucho". (VID05MO)

"Tengo a mi cargo a mi madre y a mi padre. Uno tiene 87 y mi madre 89 hace ahora..., mayorcitos los dos. Estamos ahora mi hermana y yo, entre las dos los vamos cuidando". (VID06MO)

La dedicación de las mujeres al cuidado de los demás (personas mayores e hijos pequeños) es palpable, como queda claro en estos testimonios:

"A mi niña le cuesta más, se esfuerza y, aunque le cuesta más que al hermano, es más responsable y ella apenas ha tenido niñez porque se hizo cargo de su hermano con siete años, porque ella lo que ha hecho ha sido cuidar de su hermano". (VID12MO)

"Tengo otra niña de 22 años, del colegio salió sin sacarse la ESO, la verdad es que a mí me hacía falta que me ayudara con mi madre... yo trabajaba y no se lo sacó. Ya cuando mi madre murió siguió con sus estudios, hizo un grado medio también". (VID11MO)

\section{ANÁLISIS Y DISCUSIÓN DE RESULTADOS}

A tenor de los resultados expuestos, las familias andaluzas participantes en este estudio, que viven en un ámbito rural, siguen un claro patrón de familia nuclear tradicional. La mayoría son matrimonios de hombre y mujer con una media de dos hijos; en algunos casos los abuelos cohabitan con ellos. En esta línea tradicional, destacar que son las madres entrevistadas quienes reconocen ocuparse de los familiares más mayores y de sus hijos e hijas, circunstancia constatada en otros estudios realizados en el ámbito rural andaluz (Gálvez y Matus, 2012). Se aprecia cómo, a pesar de los cambios acontecidos en las familias a nivel general, éstas siguen desempeñando un rol similar en los procesos de socialización. Collet (2013) lo expresaba con estas palabras:

...desde los años noventa se vive un proceso de metamorfosis del continente y el contenido de las familias (...) hay una creciente diversidad de formas, trayectorias y estructuras familiares que, por el contrario, no nos parece que corresponda a una diversidad en los modelos, los objetivos y las normatividades familiares a la hora de socializar a los niños (p. 84). 
Cuando en la actualidad se dice que la familia como institución social ha cambiado mucho (Meil, 2004), se suele aludir a los diferentes tipos de familia que se han hecho visibles recientemente junto a la nuclear: familias monoparentales, reconstituidas, homosexuales, etc. Según Gomila (2005):

el aumento de la ruptura e inestabilidad conyugal, la aparición de nuevos vínculos de parentesco, la entrada generalizada de la mujer en el mercado de trabajo y la redistribución de los roles sexuales en el ámbito doméstico, la diversificación de las formas familiares, etc. han supuesto una transformación de la familia y de las relaciones familiares (p. 515).

Sin embargo, esta diversidad de configuraciones familiares no se ha dado por igual en todos los contextos. Como señalan Barbadillo, Gómez, García-Borrego y Caballero (2008: 157) en referencia a Andalucía, "a grandes trazos, el hábitat rural muestra rasgos de corte tradicional y, en general, posee una población cuyas características coinciden con las que tradicionalmente han venido definiendo a los entornos rurales".

Coinciden estas palabras con los hallazgos de este estudio. A través de sus testimonios no se ha apreciado que estas familias andaluzas de ámbito rural hayan experimentado cambios notables en los últimos años. Es evidente que son las madres, trabajen fuera de casa o no, quienes se ocupan en mayor medida (o en su totalidad) de las labores domésticas, del cuidado de los mayores, del cuidado y la educación de los hijos, del contacto con los centros educativos, etc. Aunque sí se puede afirmar que son unas y otros quienes se interesan por que sus hijos e hijas culminen los estudios de formación profesional. Padres y madres apuestan porque aquellos alcancen una cualificación más elevada que la suya. Conviene recordar que estos jóvenes tienen progenitores que, en la mayoría de los casos, no han terminado la educación obligatoria. Parece un dato muy elocuente que informa que, al menos en el ámbito rural andaluz, la formación reglada no ha sido un bien común. Esta importante laguna condiciona a esta región a nivel social, cultural y económico. Quizás por las situaciones vitales que han tenido que afrontar, dibujadas aquí con pocas pinceladas, estas familias tienen muy claro que la formación de sus hijos e hijas es un objetivo irrenunciable. Se implican en la medida de lo posible en el acompañamiento en el plano académico (García-Gómez y Ordóñez-Sierra, 2016), y no escatiman para animarles y sostenerles, a fin de que el día de mañana puedan desenvolverse económicamente por sí mismos.

La principal diferencia que se aprecia en comparación con generaciones anteriores radica en el tipo de relación que mantienen estos hombres y mujeres con sus hijos e hijas, lo cual parece estar estrechamente vinculado con la década en la que nacieron. Y es que la edad media de los padres y las madres entrevistados lleva a rescatar las palabras de Meil (2006), referidas a los cambios acontecidos en las relaciones familiares a partir de la década de los sesenta, que alertan sobre una modificación del estilo educativo. Es el paso de un modelo paterno-filial más autoritario a otro más negociador:

Los jóvenes y adolescentes que comenzaron a cuestionar los estilos educativos autoritarios, desde la perspectiva actual, son los que con el tiempo han pasado a ser padres y han ido cambiando sus estilos educativos a favor de una flexibilización cada vez mayor de las normas y el establecimiento de unas relaciones cada vez más negociadas entre todos los miembros (p. 38). 
El énfasis que, según este autor, ponen madres y padres en mantener una relación de amistad con sus descendientes también se ha visto reflejado en las palabras de las personas entrevistadas, quienes no han realizado pronunciamientos autoritarios ni severos. Insistían en que sus hijos e hijas debían realizar los estudios que les gustasen en relación a la especialidad; la decisión debía ser de ellos y los estudios les tenían que proporcionar satisfacción a nivel personal.

Sí está bastante claro que el acceso al empleo es una aspiración crucial para estas familias. Como resaltan en su investigación Gradaílle, Varela y Valenzuela (2016: 58), "en una sociedad que experimenta desde hace años una crisis económica muy acentuada, el aspecto que más inquieta a las familias es el futuro que les espera a sus hijos en el ámbito laboral". Estos hombres y mujeres que abandonaron los estudios a muy temprana edad para incorporarse al mundo laboral, para contribuir al sostén de sus familias, aspiran a que el trabajo ocupe otro lugar en las vidas de sus hijos e hijas. Lo vinculan en mayor medida con la realización y el bienestar personal, anhelan que el trabajo no conlleve sufrimiento, sacrificio, explotación ni sometimiento.

A pesar de las dificultades económicas que afrontan muchas de estas familias, en sus discursos no reclaman que sus hijas e hijos abandonen los estudios para buscar cualquier tipo de trabajo que les permita contribuir a la economía doméstica. Están satisfechos de que realicen estudios de FP, y confían en que serán una vía idónea para lograr una movilidad social ascendente. Propósito cuyo logro sigue estando cuestionado en la investigación (Breen y Jonsson, 2005). El aceptar e incluso fomentar que cursen formación profesional frente al bachillerato es un dato que confirma lo que numerosos estudios han planteado: el nivel formativo de padres y madres tiene implicaciones en las trayectorias académicas de los jóvenes y en sus decisiones en relación a los estudios postobligatorios a cursar (Foley, 2007; lanelli, 2002; Rosselló y Oliver, 2014; Schnabel et al., 2002). Además, los contextos de estos jóvenes también contribuyen a trazar determinados itinerarios académicos y vitales. La escasa y dispersa oferta educativa y cultural de muchos pueblos, el descenso del número de becas (y de su montante económico), la precariedad del mercado laboral, etc., son factores que también inciden en las aspiraciones y en las expectativas de la juventud y de sus familiares.

\section{Conclusiones}

Una primera conclusión del estudio presentado es que se constata la necesidad de que las familias del alumnado de formación profesional sean sujetos de investigación. Es un colectivo al cual no es fácil acceder, pero sí es posible. Pueden aportar muchos datos que permitan conocer mejor al alumnado de FP. El contexto vital de éste es crucial para enmarcar y reconducir, si es preciso, sus trayectorias académicas así como sus aspiraciones laborales (García-Gómez y Blanco, 2015). Desde estas páginas se defiende pues la conveniencia de recabar datos sobre estas familias a nivel extensivo, que permitan realizar análisis más completos y complejos.

Para las investigadoras el contacto directo con las madres y los padres ha sido muy valioso, evidenciando las palabras de Izquierdo (2013: 14), en relación a los testimonios que aportan las personas que participan en las investigaciones: "se manifiesta 
entonces la superficialidad de la pregunta frente a la hondura del relato espontáneo". La entrega generosa que han hecho estas personas compartiendo información de sus propias vidas, de sus problemas y de sus anhelos, es de agradecer.

Se puede concluir diciendo que estas familias andaluzas que viven en un entorno rural se ocupan y preocupan de la formación de sus hijos e hijas, a pesar de las dificultades que afrontan en su día a día. Es posible que estas circunstancias contribuyan para que valoren el papel que los estudios pueden desempeñar en las vidas de sus hijos e hijas. Se ha comprobado que confían en la formación profesional, en su utilidad para que -a pesar de los problemas actuales a nivel laboral- la titulación conseguida les permita acceder a un buen empleo, les facilite el que se desarrollen como personas adultas, que puedan emanciparse y disponer de los recursos precisos para vivir dignamente. En definitiva, aspiran a que sus hijos y sus hijas disfruten de una vida mejor que las suyas, implicándose y contribuyendo en la medida de lo posible para que así sea.

\section{ReFERENCIAS BIBLIOGRÁFICAS}

Attwell, G. y Baumgartl, B. (Eds.) (2008). Creating Learning Spaces: Training and Professional Development for Trainers. Vienna: Navreme.

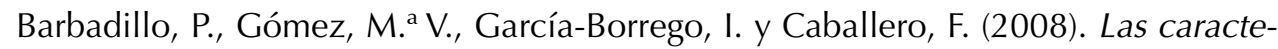
rísticas territoriales en la composición y funcionamiento de las redes familiares en Andalucía. Sevilla: Instituto de Estadística de Andalucía.

Breen, R. y Jonsson, J. O. (2005). Inequality of opportunity in comparative perspective: recent research on educational attainment and social mobility. Annual Review of Sociology, 31, 223-243. http://doi.org/10.1146/annurev.soc.31.041304.122232.

Cedefop (2011). Vocational education and training is good for you. The social benefits of VET for individuals. Research paper 17. Luxembourg: Publications Office of the European Union.

Chappell, C. (2003). Researching Vocational Education and Training: where to from here? Journal of Vocational Education and Training, 55(1), 21-32.

Collet, J. (2013). ¿Cómo y para qué educan las familias hoy? Barcelona: Icaria.

Escudero, J. M. y Martínez, B. (2012). Las políticas de lucha sobre el fracaso escolar: ¿programas especiales o cambios profundos del sistema y la educación? Revista de Educación, $\mathrm{n}^{\circ}$ extraordinario, 174-193.

Feinstein, L. y Peck, S. C. (2008). Unexpected Pathways Through Education: Why Do Some Students Not Succeed in School and What Helps Others Beat the Odds? Journal of Social Issues, 64(1), 1-20. http://doi.org/10.1111/j.1540-4560.2008.00545.x.

Flick, U. (2007). Introducción a la investigación cualitativa. Madrid: Morata.

Foley, P. (2007). The socio-economic status of vocational education and training students in Australia. Adelaide: NCVER.

Gálvez, L. y Matus, M. (2012). Género y ruralidad en Andalucía: Un diagnóstico regional. Revista de Estudios Regionales, 94, 195-218.

García-Gómez, S. y Blanco, N. (2015). Los Ciclos Formativos de Grado Medio: una opción para salir del laberinto personal y académico. Tendencias Pedagógicas, 25, 301-320. 
García-Gómez, S. y Ordóñez-Sierra. R. (2016). La implicación educativa de familias de alumnado de formación profesional. RASE. Revista de la Asociación Española de Sociología, 9(3), 373-385.

García-Gómez, S., Ordóñez-Sierra, R., Vinuesa, E. y Izquierdo, R. (2016). Expectativas de las familias del alumnado de formación profesional acerca de su futuro laboral. Archivos Analíticos de Políticas Educativas, 24(117), 1-28. http://doi. org/10.14507/epaa.24.2484.

García, M., Casal, J., Merino, R. y Sánchez, A. (2013). Itinerarios de abandono escolar y transiciones tras la enseñanza secundaria obligatoria. Revista de Educación, 361, 65-94. http://doi.org/10.4438/1988-592X-RE-2011-361-135.

Gessler, M. y Freund, L. (Eds.) (2015). Crossing Boundaries in Vocational Education and Training: Innovative Concepts for the 21st Century. Bremen: University of Bremen.

Gomila, M. A. (2005). Las relaciones intergeneracionales en el marco de la familia contemporánea: Cambios y continuidades en transición hacia una nueva concepción de la familia. Historia Contemporánea, 31, 505-542.

Gradaílle, R., Varela, L. y Valenzuela, A. (2016). Preocupaciones del profesorado y de las familias sobre los tiempos escolares y de ocio del alumnado de Educación Secundaria Postobligatoria. Revista Interuniversitaria de Formación del Profesorado, 86(30.2), 49-62.

Greinet, W-D. (2004). Los "sistemas" europeos de formación profesional: algunas reflexiones sobre el contexto teórico de su evolución histórica. Revista Europea de Formación Profesional, 32, 18-26.

Homs, O. (2008). La formación profesional en España. Hacia la sociedad del conocimiento. Barcelona: Fundación la Caixa.

Iannelli, C. (2002). Parental Education and Young People's Educational and Labour Market Outcomes: A Comparison across Europe. Working Papers 45. Mannheim.

Izquierdo, R. (2013). Diario de campo. Madrid: Caballo de Troya.

Kvale, S. (2011). Las entrevistas en la investigación cualitativa. Madrid: Morata.

LOMCE (2013). Ley Orgánica para la Mejora de la Calidad de la Educación, de 10 de diciembre de 2013. Recuperado de http://www.boe.es/diario_boe/txt. php?id=BOE-A-2013-12886.

Lorente, A. (2006). Cultura docente y organización escolar en los institutos de secundaria. Profesorado. Revista de currículum y formación del profesorado, 10(2), 1-13.

Marhuenda-Fluixá, F., Salvá, F., Navas, A. A. y Abiétar, M. (2015). Twenty Years of Basic Vocational Education Provision in Spain: Changes and Trends. International Journal for Research in Vocational Education and Training, 2(2), 137-151. http:// doi.org/10.13152/IJRVET.2.2.8.

Martínez, M. ${ }^{\mathrm{a} J .}$ (2001). ¿Hacia la consolidación de un proyecto europeo?: problemas, obstáculos e inconvenientes de la Formación profesional en la Unión Europea. Revista Complutense de Educación, 12(1), 251-294.

MECD (2016). Datos y cifras. Curso escolar 2016/2017. Madrid: Secretaría General Técnica. Recuperado de http://www.mecd.gob.es/servicios-al-ciudadano-mecd/ $\mathrm{dms} / \mathrm{mecd} /$ servicios-al-ciudadano-mecd/estadisticas/educacion/indicadorespublicaciones-sintesis/datos-cifras/Datosycifras1617esp.pdf. 
Meil, G. (2004). La familia española en el contexto de la Unión Europea. Arbor CLXXVIII, 702, 421-449.

Meil, G. (2006). Padres e hijos en la España actual. Barcelona: Fundación la Caixa.

Merino, R. (2005). Apuntes de historia de la formación profesional reglada en España. Algunas reflexiones para la situación actual. Témpora, 8, 211-236.

Misra, P. K. (2011). VET teachers in Europe: policies, practices and challenges. Journal of Vocational Education \& Training, 63(1), 27-45.

Planas, J. (2012). ¿Qué es y para qué sirve hoy la formación profesional? De la VT (formación profesional) a la VET (formación y educación profesional). RASE. Revista de la Asociación de Sociología de la Educación, 5(1), 5-15.

Psifidou, I. (2014). Evolución histórica de la Formación Profesional en Europa. Historia de la Educación, 33, 353-359.

Renés, P. y Castro, A. (2013). Análisis de la situación de la formación profesional desde el punto de vista de sus protagonistas. Educatio Siglo XXI, 31(2), 255-276.

Rodríguez, G. Gil, J. y García, E. (1996). Metodología de la investigación cualitativa. Archidona: Aljibe.

Rosselló, J. y Oliver, X. (2014). The Determinants of Non-compulsory Education Demand: An Analysis from the Students' Perspective. Revista de Educación, 365, 202-236. http://doi.org/10.4438/1988-592X-RE-2014-365-270.

Schnabel, K. U., Alfeld, C., Eccles, J. S., Köller, O. y Baumert, J. (2002). Parental Influence on Students' Educational Choices in the United States and Germany: Different Ramifications-Same Effect? Journal of Vocational Behavior, 60, 178-198. http://doi.org/10.1006/jvbe.2001.1863. 